\title{
Die Rechtsordnung im nachrevolutionären Nicaragua: Anspruch und Wirklichkeit
}

von Marta Patricia Baltodano, Reinhard Junghanns, Michael Sommer

\section{Vorgeschichte}

Der Sturz der seit 45 Jahren regierenden Familiendiktatur der Somozas am 19. Juli 1979 leitete eine tiefgreifende revolutionäre Umwälzung der nicaraguanischen Rechtsordnung ein. Mit der Verkündung und Inkraftsetzung der neuen nicaraguanischen Verfassung am 9. Januar $1987^{1}$ wurde dieser Prozeß institutionalisiert und gelangte zu einem vorläufigen Abschluß. Die neue Verfassung schuf den Rahmen, in dem sich - logisch und konsequent - die Politik der ersten siebeneinhalb Revolutionsjahre wiederfindet.

Bereits am 16. Juni 1979, also etwa einen Monat vor seiner Machtergreifung, verpflichtete sich der Frente Sandinista de Liberación Nacionál (FSLN) in der ersten öffentlichen Bekanntmachung der von ihm angekündigten Regierung des Nationalen Wiederaufbaus dazu, "die Durchführung eines Plans des nationalen Wiederaufbaus in die Wege zu leiten, dessen fundamentales Ziel darin besteht, ein wahrhaft demokratisches System in Nicaragua zu errichten ...". ${ }^{2}$

Diese Proklamation stellt zwar kein Gesetz dar, ihr kommt aber dennoch eine gewisse Verbindlichkeit zu, da es sich hierbei um die erste, von den Sandinisten unterzeichnete Erklärung handelt, mit der sie sich gegenüber befreundeten Nationen zur Errichtung eines demokratischen Systems in Nicaragua verpflichtet haben. In dem Programm wird eine für die Errichtung eines wahrhaft demokratischen Systems erforderliche Gesetzgebung angekündigt, beruhend auf den Prinzipien der Gerechtigkeit und des sozialen Fortschritts. Außerdem sollen im vollen Umfange das Recht aller Nicaraguaner auf Teilnahme am politischen Leben und auf allgemeine Wahlen gewährleistet und die Organisation und die Arbeit von politischen Parteien garantiert werden.

Die Verantwortung für die exekutive Gewalt und für die Administration des Staates sollte bei der Regierungsjunta liegen. Es wurde vorgesehen, einen Staatsrat zu bilden, der die gesetzgeberischen Aufgaben mit der Regierungsjunta teilt. Als oberste Justizbehörde sollte der Oberste Gerichtshof eingerichtet werden und es sollte sichergestellt werden, daß die rechtsprechende Gewalt allein für die Jurisdiktion zuständig sein sollte und daß ihre Mitglieder die für die Ausübung ihrer Funktionen erforderliche Befähigung und Unvoreingenommenheit aufwiesen. Des weiteren sollte durch die rechtsprechende Gewalt

1 Im Gesetzesblatt LA GACETA, Jahrgang XCI, No. 5 am 9. Januar 1987 publiziert.

2 Textabdruck z. B. EL DIA, México v. 26. 6. 1979; NOTICIAS' DE GUATEMALA 1 (1979) Nr. 21 v. 16.7. 1979; vgl. dazu im übrigen Douglas W. Payne, The Democratic Mask, New York 1985, S. 20. 
die korrekte Rechtsanwendung wiederhergestellt und die Wahrnehmung der bürgerlichen Rechte in vollem Umfang garantiert werden.

An diesen, der Machtergreifung der Sandinisten vorausgegangenen Texten, die lediglich Entwürfe für eine mögliche politische und rechtliche Struktur der neuen nicaraguanischen Rechtsordnung waren, orientierten sich die ersten nach dem Umsturz verabschiedeten Gesetze.

\section{Das Grundsatzstatut vom 20. Juli 1979}

Unmittelbar nach ihrer Machtergreifung erließ die Regierungsjunta am 20. Juli 1979 das Grundsatzstatut (Estatuto Fundamental).

$\mathrm{Zu}$ den wichtigsten Bestimmungen gehörte die Aufhebung der vorherigen Staatsverfassung und der Verfassungsgesetze, die Auflösung des Abgeordnetenhauses und des Senats sowie die Auflösung des Obersten Gerichtshofes, der Appellationsgerichte und des Obersten Arbeitsgerichts.

Gleichzeitig legte das Statut die drei Staatsgewalten fest:

- die Regierungsjunta,

- der Staatsrat,

- die Gerichtshöfe. ${ }^{3}$

Die Regierungsjunta des Nationalen Wiederaufbaus übernahm die gesamten Befugnisse der ausführenden Gewalt, teilte sich die Befugnisse der gesetzgeberischen Gewalt mit dem Staatsrat und übertrug die rechtsprechende Gewalt dem Obersten Gerichtshof und den übrigen Gerichten des Landes. Die Vertreter der beiden ersten Staatsorgane waren bereits vor der Verkündung ernannt worden. ${ }^{4}$ Dagegen stand die Berufung der sieben

3 Artikel 9 des Grundsatzstatuts, publiziert in der Gesetzessammlung von Lacayo, Rolando D./Laçayo de Arauz, Matha (Hrsg.): Decretos, Leyes para Gobierno de un país a través de una Junta de Gobierno de Reconstrucción Nacional, Band I, Managua 1979, S. 1 ff.; Anm.: Úbersetzungen von Gesetzestexten stammen von den Verfassern.

4 Die Regierungsjunta des Nationalen Wiederaufbaus bestand zunächst aus fünf Mitgliedern:

Daniel Ortega Saavedra, Sergio Ramírez Mercado und Moisés Hassán Morales als Vertreter des FR ENTE SANDINISTA DE LIBERACION NACIONAL (FSLN) sowie Violeta Barrios de Chamorro und Alfonso Robelo Callejas als Vertreter der bürgerlichen Gruppen, die die Revolution unterstützt hatten. Die Zusammensetzung des Staatsrates, die vorher im Regierungsprogramm festgelegt worden war, wurde im Jahre 1980 einseitig von der FSLN-Mehrheit in der Regierungsjunta geändert. Die ursprüngliche Mitgliederzahl des Staatsrates wurde von 33 auf 51 erhöht, von denen 39 Mitglieder in sandinistischen oder diesen nahestehenden Organisationen waren. Violeta Barrios de Chamorro und Alfonso Robelo Callejas erklärten daraufhin ihren Rücktritt aus der Junta. Sie wurden durch den ehemaligen Präsidenten der Zentralbank, Arturo Cruz Porras, und den der konservativen Partei angehörenden Rechtsanwalt Rafael Córdova Rivas ersetzt.

Alfonso Robelo Callejas wirkte noch bis April 1982 als Oppositionsführer seines 1978 gegründeten MOVIMIENTO DEMOCRATICO NICARAGUENSE (MDN), bevor er nach schweren Úbergriffen mit der gesamten Parteiführung nach Costa Rica ins Exil ging. Nachdem auch der Konservative Cruz 1981 aus der Junta ausschied und als Botschafter nach Washington ging, wurde die Junta auf drei Mitglieder verkleinert. Hassán, der ebenfalls ausschied, wurde 1985 Bürgermeister von Managua. Nach der Wahl vom November 1984 übernahmen die vormaligen Juntamitglieder Daniel Ortega Saavedra und Sergio Ramírez Mercado am 10. Januar 1985 ihre neuen Funktionen als Staatspräsident bzw. Vize-Präsident auf sechs Jahre. 
Richter des Obersten Gerichtshofes, der sechs Appellationsgerichte und des Obersten Arbeitsgerichtes noch aus. Für ihre Ernennung war die Regierungsjunta zuständig. Nach der Ernennung dieser Richter ernannte der Oberste Gerichtshof in den folgenden Tagen 34 Bezirksrichter und 150 Amtsrichter. ${ }^{5}$

Gemäß dem Grundsatzstatut gab es zwei Gesetzgebungsverfahren:

a) In Ausübung ihrer gesetzgeberischen Befugnisse arbeitete die Regierungsjunta Gesetze aus, die dem Staatsrat zur Kenntnis vorgelegt wurden. Der Staatsrat hatte dann das Recht, dagegen Einspruch einzulegen, und zwar innerhalb einer Frist von fünf Tagen und mit einer Mehrheit von zwei Drittel der Stimmen seiner Mitglieder. Andernfalls würden die Gesetze verkündet werden. ${ }^{6}$

b) Der Staatsrat hatte die Möglichkeit, der Regierungsjunta eine mit Stimmenmehrheit gebilligte Gesetzesinitiative vorzulegen. Falls die Junta Abänderungen vornahm, wurde der Entwurf wieder an den Staatsrat zurückgesandt, der dann in der für das andere Verfahren festgelegten Weise sein Recht auf Einspruch geltend machen konnte.

Ebenso wurde verfügt, daß das Grundsatzstatut von der Regierungsjunta oder mit einer besonderen mehrheitlichen Initiative des Staatsrates (zwei Drittel der Stimmen) teilweise oder vollständig abgeändert werden konnte, und zwar nach dem gleichen Verfahren, wie es bei einem gewöhnlichen Gesetz zur Anwendung kam.

\section{Die ersten Verfassungsgesetze}

Formell stellte das Grundsatzstatut keine Staatsverfassung dar. Aufgrund des Verfahrens, durch das es verkündet wurde und durch das es abgeändert werden konnte, stand es auf einer Ebene mit dem gewöhnlichen Gesetz und besaß damit keinen höheren Rang als alle folgenden, von der Regierungsjunta erlassenen Gesetze. Wegen seines die Staatsorganisation regelnden Inhalts hat man jedoch in der Praxis das Grundsatzstatut zusammen mit dem am 21. August 1979 verkündeten Statut über die Rechte und Garantien der Nicaraguaner ${ }^{7}$ als die erste Verfassung der Revolution betrachtet. Mit den Bestimmungen über die Staatsorganisation, die Rechte und Garantien der Staatsbürger und des

Vgl. dazu insbesondere Christian, Shirley: Nicaragua-Revolution in the Family, New York 1985, S. $147 \mathrm{ff}$.; zur Entwicklung aus sozio-politischer Sicht vgl. Wolf, Ulrike: Soziopolitische Konflikte in Nicaragua 1979-1982, Saarbrücken/Fort Lauderdale 1985.

5 Lawyers Committee for International Human Rights (Hrsg.): Nicaragua-Revolutionary Justice. A Report on Human rights and the Judicial System, New York/Washington D.C., April 1985, S. 16.

6 Artikel 14 Grundsatzstatut vom 20. Juli 1979; diese Einspruchsmöglichkeit des Staatsrates wurde am 2. Mai 1980 durch Dekret Nr. 388 beseitigt, vgl. Lawyers Committee for International Human Rights (Anm. 5), S. 15 Fn. 2.

7 Vgl. deutsche Ubersetzung in: Nicaragua-Gesellschaft e.V. ( qrsg.): Dekrete und Bestimmungen der gegenwärtigen Gesetzgebung in Nicaragua, die gegen die Menschenrechte verstoßen, Nicaragua - Stimmen der Freiheit, Heft 2, Bonn 1985, 3. Aufl., S. 33 ff. Diese Publikation ist die deutsche Úbersetzung von: Comisión Permanente de Derechos Humanos ( $\mathrm{CPDH})$ : Decretos y Disposiciones de la actual legislación nicaragüense que atentan contra los derechos humanos $3^{\circ}$ Edición Actualizada, Managua, Junio 1984. 
geltenden Rechtssystems enthielten diese beiden Texte das materielle Verfassungsrecht des revolutionären Staates vor der jetzt verkündeten neuen Verfassung. Aufgrund der Tatsache jedoch, daß diese beiden Statuten rechtlich nicht den Rang einer Verfassung hatten, wurden in der Folgezeit häufig Gesetze erlassen, die gewollt im Widerspruch zu den Bestimmungen der beiden Statuten standen (lex posterior-Regel).

Das Statut über Rechte und Garantien der Nicaraguaner vom 21. August 1979 regelte in Teil I die Rechte des Volkes, in Teil II die bürgerlichen und politischen Individualrechte und in Teil III Bestimmungen über wirtschaftliche, soziale und kulturelle Einzelrechte. Kapitel II Art. 6 des Grundsatzstatuts enthielt auch eine Regelung über die auf internationaler Ebene völkerrechtlich geschützten Menschenrechte. Artikel 6 lautet:

"Gewährleistet wird die volle Gültigkeit der in der allgemeinen Erklärung der Menschenrechte, dem Internationalen Pakt über wirtschaftliche, soziale und kulturelle Rechte, dem Internationalen Pakt über bürgerliche und politische Rechte der Vereinten Nationen und der Amerikanischen Erklärung über die Rechte und Pflichten des Menschen verankerten Grundrechte, und zwar in der Form, wie sie im Statut über die Rechte und Garantien der Nicaraguaner niedergelegt ist, das gleichzeitig mit diesem

Statut erlassen wird."

Da durch einen Regierungswechsel in einem Land die Rechtskontinuität hinsichtlich seiner völkerrechtlichen Verpflichtungen nicht unterbrochen wird, hatte die Bestimmung des Artikel 6 des Grundsatzstatuts lediglich feststellenden Charakter, soweit bereits von der vorangegangenen Regierung ratifizierte internationale Konventionen angesprochen waren. ${ }^{8}$

Die Formulierung des Artikel 6 des Grundsatzstatuts, die von einer Verbürgung der Grundrechte in der Form sprach, "wie sie im Statut über die Rechte und Garantien der Nicaraguaner niedergelegt ist ", machte jedoch bereits deutlich, daß die Regierung nicht bereit war, die in den internationalen Konventionen enthaltenen Bestimmungen mehr als nur relativ, das heißt bezogen auf das, was im staatlichen Gesetz garantiert wird, zu erfüllen. Eine solche Absicht der Regierung kommt dann auch in der Tatsache zum Ausdruck, daß einige der betreffenden Rechtsbegriffe im Statut über die Rechte und Garantien nicht nur abgeändert, sondern auch umgebildet worden sind und zum Teil sogar vollständig fehlen. Die nicaraguanische Regierung ratifizierte sodann die Amerikanische Menschenrechtskonvention und erhob sie durch Dekret Nr. 174 vom 23. November 1979 ausdrücklich in den Rang eines "Staatsgesetzes".9 Nach der lex posterior-Regel müßten die Bestimmungen des Statuts über die Rechte und Garantien der Nicaraguaner, die der Amerikanischen Menschenrechtskonvention zuwiderliefen, damit aufgehoben bzw. abgeändert worden sein. Dies müßte auch für den umstrittenen Artikel 51 des Statuts gelten, der die Ausübung der im Statut festgelegten Rechte und Garantien für

8 Vgl. dazu die Auffassung der COMISION PERMANENTE DE DERECHOS HUMANOS (CPDH) in: Nicaragua-Gessellschaft e.V. (Anm 7), S. 8 f.

9 Lawyers Committee for International Human Rights (Anm. 5), S. 17; Nicaragua-Gesellschaft e.V. (Hrsg.) (Anm. 7), S. 8. 
diejenigen Personen für den Zeitraum von 60 Tagen außer Kraft setzte, gegen die aufgrund ihrer während des Somoza-Regimes begangenen Verbrechen, die nach dem nationalen Strafgesetzbuch und den internationalen Ubereinkommen strafbar sind, ein Ermittlungsverfahren lief. ${ }^{10}$ Dieser Artikel ist von den Juristen der unabhängigen Comisión Permanente de Derechos Humanos (CPDH) als fast vollständige Außerkraftsetzung der Menschenrechte kritisiert worden. ${ }^{11}$ Am 12. März 1980 wurde der Internationale Pakt über bürgerliche und politische Rechte ratifiziert. ${ }^{12}$

\section{Die Notstandsgesetze und das Gesetz über die Aufrechterhaltung der öffentlichen Ord- nung und Sicherheit}

Mit Dekret Nr. 10 vom 22. Juli 1979 wurde über das Land der Staatsnotstand verhängt. Dabei wurden die Rechte und Garantien all derer aufgehoben, gegen die in Strafsachen ermittelt wurde, die sie zur Zeit des Somoza-Regimes begangen haben sollten. Dieses Dekret und das Gesetz über die Aufrechterhaltung der öffentlichen Ordnung und Sicherheit ergänzten sich gegenseitig. Letzteres wurde mit Dekret Nr. 5 vom 20. Juli 1979 durch die Regierungsjunta verkündet. Das Dekret, das einen der Hauptpfeiler darstellt, auf den sich die Existenz der Volksgerichte stützt, sollte für die Dauer des Staatsnotstandes gelten. ${ }^{13}$ Das Gesetz über die Aufrechterhaltung der öffentlichen Ordnung und Sicherheit war seinerzeit nach weitverbreiteter Auffassung gerechtfertigt, weil es auf die Wiederherstellung der öffentlichen Ordnung abzielte. Obwohl viele seiner Tatbestände bereits im gewöhnlichen Strafrecht vorgesehen waren, verfügte es die Einrichtung von "besonderen Notstandsgerichten" (Art. 5), die in einem summarischen Verfahren mit wenigen verfahrensrechtlichen Garantien über Verstöße gegen die Bestimmungen dieses Gesetzes entscheiden sollten. ${ }^{14}$ Diese Gerichte bestanden aus jeweils drei von den Regierungsbehörden jeder Bezirkshauptstadt ernannten Richtern. ${ }^{15}$ Diese Sondergerichte haben, einem Bericht der Internationalen Juristenkommission zufolge, niemals wirklich

10 Die Maßnahme des Art. 51 des Statuts über die Rechte und Garantien der Nicaraguaner ist mit Dekret Nr. 116 vom 20. Oktober 1979 um weitere sechs Monate verlängert worden.

11 Vgl. Nicaragua-Gesellschaft e.V. (Hrsg.) (Anm. 7), S. 7.

12 Auch das Fakultativprotokoll zu diesem Pakt und der Internationale Pakt über wirtschaftliche, soziale und kulturelle Rechte wurden von Nicaragua ratifiziert.

13 Vgl. Amnesty International (Hrsg.): Nicaragua-The Human Rights Record, London, March 1986, im folgenden wird jeweils auf die deutsche Úbersetzung verwiesen, die zwar nicht im AI-Publikationsverzeichnis aufgeführt, aber bei der deutschen Sektion auf Anfrage erhältlich ist, hier: S. 4 ff., $18 \mathrm{ff}$.

14 Dieses Verfahren bestand aus den folgenden Schritten:

a) Nach Erstattung der Anzeige wurde der Angeklagte auf schriftlichem oder mündlichem Wege davon unterrichtet. Er hatte dann 48 Stunden Zeit, um darauf zu antworten.

b) Unmittelbar danach wurde das Verfahren zur Beweisaufnahme eröffnet, das sich über drei Tage erstreckte.

c) Nach Ablauf dieser Frist verkündete das Gericht innerhalb von 48 Stunden das Urteil.

Die Beurteilung der Beweise durch den Richter erfolgte nach dem Prinzip des "gesunden Menschenverstandes", nicht aber nach den Regeln des Strengbeweises, Dekret 195 vom 5. Dezember 1979.

15 Nicaragua-Gesellschaft e.V. (Hrsg.) (Anm. 7), S. 16 
funktioniert. Weitere Sondergerichte sowie eine Sonderstaatsanwaltschaft wurden durch die Dekrete Nr. 185 und Nr. 186 vom 29. November 1979 eingesetzt. Diese Gesetze verstoßen nach Ansicht der Internationalen Juristenkommission gegen die Menschenrechte. In ihrem Bericht heißt es: "Diese Sondergerichte sind Ausnahmegerichte, in denen sich eine politische Justiz den Anschein der Rechtmäßigkeit verleiht und außerhalb der Grundsätze des demokratischen Strafprozesses agiert. Diese Gerichte sind immer antidemokratisch und verstoßen gegen das Prinzip des gesetzlichen Richters. "16

Sowohl die Einführung der besonderen Notstandsgerichte als auch einzelne Vorschriften mit unpräzisen und dehnbaren Formulierungen sind angreifbar. So wird nach Art. 4 Abs. c des Gesetzes "mit öffentlichen Arbeiten (Arrest) von drei Monaten bis zu zwei Jahren bestraft, wer . . . die mündliche oder schriftliche Verbreitung von Erklärungen, Aufrufen oder Manifesten, die darauf gerichtet sind, den Volksinteressen zu schaden und die vom Volk erzielten Errungenschaften wieder rückgängig zu machen“. Die Internationale Juristenkommission hat diese Bestimmung kritisiert ${ }^{17}$ und darauf hingewiesen, daß das Gesetz in verschiedenen weiteren Passagen gegen den Bestimmtheitsgrundsatz verstößt.

Vier Monate nach seiner Verkündigung wurde das Gesetz über die öffentliche Ordnung und Sicherheit von der Regierung abgeändert. ${ }^{18}$ Dabei wurde verfügt, daß die Ahndung von Verstößen gegen dieses Gesetz der ordentlichen Gerichtsbarkeit zufallen solle, jedoch unter Beibehaltung des summarischen Verfahrens, wie es bereits für die Notstandsgerichte vorgesehen war.

Am 9. August 1980 wurde per Dekret Nr. 488 das Gesetz über die Aufrechterhaltung der öffentlichen Ordnung und Sicherheit erneut abgeändert. Der neue Art. 4 Abs. c erhielt danach folgenden Wortlaut:

"Mit Arrest und öffentlichen Arbeiten von zehn Tagen bis zu zwei Jahren wird bestraft, wer die folgenden Delikte begeht: ... c) Die mündliche oder schriftliche Verbreitung von Erklärungen, Aufrufen oder Manifesten, mit denen anzugreifen versucht wird:

1. die nationale Sicherheit und Einheit, die öffentliche Sicherheit und die Volkswirtschaft,

2. der Schutz der Ordnung und die Verhütung von Straftaten,

3. der Schutz der Volksgesundheit, der Sittlichkeit, der Würde der Personen, das Ansehen und die Rechte anderer,

4. die Autorität und Unabhängigkeit der richterlichen Gewalt."

Zugleich wurde die Konfiszierung des Vermögens derjenigen Angeklagten angeordnet, die wegen Uberschreitung dieses Gesetzes zu einer Strafe von mehr als fünf Jahren verurteilt wurden. ${ }^{19}$

16 Vertraulicher Bericht der Internationalen Juristenkommission an die Regierung des Nationalen Wiederaufbaus, August 1980, zit. nach Nicaragua-Gesellschaft e.V. (Hrsg.) (Anm. 7), S. 16; vgl. hierzu auch Lawyers Committee for International Human Rights (Anm. 5), S. 33.

17 Vgl. Nicaragua-Gesellschaft e. V. (Hrsg.) (Anm. 7), S. 16.

18 Dekret 148 vom 9. November 1979.

19 Die Regierungsjunta hat in den Jahren nach dem Sturz der Diktatur eine Vielzahl von Gesetzen, die in das 
Zuvor war durch Dekret Nr. 383 vom 29. April 1980 das Gesetz über den Staatsnotstand aufgehoben worden. Das Gesetz über die Aufrechterhaltung der öffentlichen Ordnung und Sicherheit und das dazugehörige gerichtliche Verfahren erhielten jedoch dauerhafte Rechtsgeltung und wurden auf diese Weise in die ordentliche Strafgesetzgebung aufgenommen. Ein Ausnahmegesetz wurde so in ein ordentliches Gesetz verwandelt. Auf diese Weise wurden eine Reihe von Verletzungen der Justizgrundrechte legalisiert, von denen hunderte von Nicaraguanern betroffen waren.

Am 9. September 1981 verhängte die Regierung per Dekret Nr. 812 für den Zeitraum von einem Jahr den wirtschaftlichen und sozialen Notstand. Rechtsgrundlage waren Art. 49 des Statuts über die Rechte und Garantien der Nicaraguaner und Art. 28 des Staatsratsstatuts. ${ }^{20}$ Mit diesem neuen Gesetz wurden eine Reihe wirtschafts- und landwirtschaftsbezogener Straftatbestände neu geschaffen. Die Wahrnehmung des Arbeitsrechts wurde eingeschränkt, und das Rechtsmittel der Beschwerde gegen die in der Folge dieses Gesetzes erlassenen Verwaltungsakte wurde aufgehoben. Die Entscheidung über Verstöße gegen dieses Notstandsgesetz oblag den ordentlichen Gerichten, wurde aber auf der Grundlage des für das Gesetz über die Aufrechterhaltung der öffentlichen Ordnung und Sicherheit festgelegten summarischen Verfahrens gefällt. Viele der durch dieses Gesetz neu eingeführten Straftatbestände waren bereits im geltenden Strafgesetzbuch vorgesehen. Dies bedeutete eine Verdopplung der Straftatbestände, ein Faktum, das willkürliche Ubergriffe erleichterte.

Zudem stand die Aufhebung des Rechtsbehelfs der Beschwerde (Art. 5 des Gesetzes) im Widerspruch zu Art. 27 Abs. 2 der Amerikanischen Menschenrechtskonvention, demzufolge die für den Schutz der Menschenrechte unerläßlichen Garantien nicht aufgehoben werden dürfen. Das Rechtsmittel der Beschwerde wird in Art. 25 dieser Konvention definiert und ist dort unter dem Titel "Rechtsschutz" aufgeführt, das heißt, es wird zu den Garantien gezählt, die unaufhebbar sind. ${ }^{21}$

Recht auf Eigentum eingreifen und in denen die Enteignung zum Teil Strafcharakter hat, erlassen; zu ihnen gehören Dekret 3 vom 20. Juli 1979 (Enteignung der Somoza-Familie und ihrer Anhänger), Dekret 38 vom 8. August 1979 (Erweiterung auf "alle dem Somozismus angehörenden Personen"), aufgehoben durch Dekret 172 vom 21. November 1979, Dekret 282 vom 7. Februar 1980 (rechtliche Situation von Personen, deren Vermögen beschlagnahmt oder untersucht wurde), Dekret 422 vom 31. Mai 1980 (Úbertragung anhängiger Fälle an die ordentlichen Gerichte), Dekret 330 vom 29. Februar 1980 (Gesetz zur Vermeidung und zur Bekämpfung der wirtschaftlichen Kapitalabwanderung), Dekret 759 vom 19. Juli 1981 (Enteignung von 15 Privatunternehmen), Dekret 805 vom 28. August 1981 (Gesetz über die Kapitalflucht), Dekret 760 vom 19. Juli 1981 (Enteignung bei mehr als sechsmonatigem Auslandsaufenthalt), Dekret 763 vom 19. Juli 1981 Enteignung bei langjährigen Freiheitsstrafen), Art. 17 des Agrarreformgesetzes vom 19. Juli 1981 (Enteignung von verlassenem Grundeigentum). Ein vom oppositionellen PARTIDO LIBERAL INDEPENDIENTE (PLI) im Juni 1986 in der Nationalversammlung eingebrachter und von Teilen des PARTIDO CONSERVADOR DEMOCRATA DE NICARAGUA (PCDN) unterstützter Antrag auf Aufhebung des Dekrets 760 vom 19. 7. 1981 wurde mit 55 gegen 18 Stimmen bei 3 Enthaltungen zurückgewiesen, vgl. Informationsdienst Nicaragua (IDN): 14/86 vom 5. 7. 1986; LA PRENSA vom 4. 6. 1986.

20 Dekret 388 vom 2. Mai 1980; vgl. Nicaragua-Gesellschaft e.V. (Hrsg.) (Anm.7), S. 25; Amnesty international (Hrsg.) (Anm. 13): S. 5.

21 Art. 25 der Amerikanischen Menschenrechtskonvention lautet: "Jede Person hat das Recht auf eine einfache und schnelle Beschwerde oder auf jedes andere gegenüber den zuständigen Richtern oder Gerichten wirksame 
Mit der erneuten Verhängung des Staatsnotstands vom 15. März 1982 (Dekret Nr. 996) ist der wirtschaftliche und soziale Notstand wieder aufgehoben worden.

Im Dezem ber 1981 erfolgte eine erneute Abänderung des Gesetzes über die Aufrechterhaltung der öffentlichen Ordnung und Sicherheit. ${ }^{22}$ Sie betraf das Verfahren, in dem über Verstöße gegen dieses Gesetz entschieden werden sollte. Die Änderungen bestanden in der Erweiterung der Frist zur Beweisaufnahme von drei auf acht Tage, die um weitere vier Tage verlängert werden konnte, und in der Verlängerung der bis zur Verkündung des Urteils zur Verfügung stehenden Frist von zwei auf drei Tage. Die Rechtsmittel der Beschwerde und Revision blieben dabei in Geltung und sollten auf ordentlichem Wege abgehandelt werden.

Durch Dekret Nr. 1074 erfolgte im Juli 1982 eine vollständige Neuregelung des Gesetzes über die Aufrechterhaltung der öffentlichen Ordnung und Sicherheit. Dabei legte man fest, welche Verhaltensweisen als Verstöße gegen die Sicherheit des Staates betrachtet werden sollten und fügte dem Gesetz neue Vorschriften hinzu, die sich auf Gewalttaten bezogen. Bei der ersten Kategorie von Delikten wurde die Höchststrafe von zehn auf dreißig Jahre erhöht. Weiterhin wurden in dieses Gesetz die ehemaligen Bestimmungen des wirtschaftlichen und sozialen Notstands als neue Straftatbestände aufgenommen. Diese bezogen sich auf Aktivitäten, die für die Wirtschaft von Belang waren, wie die Zerstörung von Rohstoffen und landwirtschaftlichen Produkten, die Verbreitung falscher Nachrichten mit negativem Einfluß auf die Volkswirtschaft, den Ankauf zu Hortungszwecken, die Spekulation sowie die "Anstiftung ausländischer Regierungen und internationaler Kreditinstitute zu Entscheidungen, die für die Volkswirtschaft von Schaden sind “. ${ }^{23}$ Art. 4 Abs. b dieses Gesetzes wurde wie folgt abgeändert:

"Mit einer Strafe von zehn Tagen bis zu zwei Jahren Arrest oder öffentlichen Arbeiten wird bestraft, wer die folgenden Delikte begeht: ...

b) mündliche oder schriftliche Verbreitung von Problemen oder Manifesten, die einen Angriff darstellen auf: ... .

4) die gesetzmäßig gebildeten Autoritäten."

Wegen seiner unpräzisen Formulierung, die zu Verfolgungsmaßnahmen gegen politische Gegner führen kann, ist auch dieser Artikel angreifbar. ${ }^{24}$

Rechtsmittel, das sie vor Verletzungen ihrer in der Verfassung, im Gesetz oder in der vorliegenden Konvention verbürgten Grundrechte schützt. Das gilt auch dann, wenn eine solche Verletzung durch Personen erfolgt, die in Erfüllung ihrer Dienstpflicht handeln."

22 Dekret 896 vom 4. Dezember 1981 (Verfahrensgesetz für Delikte gegen die Aufrechterhaltung der öffentlichen Ordnung und Sicherheit).

23 Die wirtschaftlichen Maßnahmen, die in der Abänderung des Gesetzes über die öffentliche Ordnung und $\mathrm{Si}$ cherheit mit einbezogen wurden, waren in Art. 3 Abs. b, c, e und h des Dekrets 812 des wirtschaftlichen und sozialen Notstands vorgesehen, der per Dekret 996 vom 15. März 1982 außer Kraft gesetzt worden war.

24 Amnesty International (Hrsg.) (Anm. 13), S. 19:

"Amnesty International war auch über die Definitionen von Verbrechen in dem Gesetz besorgt, besonders in Bezug a uf Verschwörung und Freiheit der Meinungsäußerung. Diese Definitionen waren ungenau und offen für willkürliche Auslegung. Sie lieferten so eine Basis für die Verfolgung von Individuen, die, während sie gegen die Regierung opponierten, weder Gewalt angewandt noch befürwortet hatten. Von besonderer Sorge war Artikel 4." 
Eine weitere Neuerung dieses Gesetzes besteht darin, daß zum Zwecke der Konfiszierung des Vermögens von Angeklagten - vorgesehen für diejenigen, die zu mehr als fünf Jahren Haft verurteilt werden - "gesetzlich vermutet wird, daß die Handlungen und Verträge, die von dem Angeklagten oder seinem Bevollmächtigten nach dem 19. Juli 1979 abgewickelt worden sind, Scheinhandlungen oder Scheinverträge sind". Gegen diese Vermutung kann man Gegenbeweise von Drittbesitzern vorlegen. Die Beurteilung dieser Beweise durch das Justizministerium erfolgt jedoch nach dem Prinzip des "gesunden Menschenverstandes $"$. ${ }^{25}$

\section{Der erneute Staatsnotstand seit 1982}

Wie erwähnt, hob die Regierung den am 22. Juli 1979 verhängten Staatsnotstand am 29. 4. 1980 wieder auf. Er wurde dann jedoch erneut am 15. März 1982 verhängt. Dabei wurden alle Rechte der Nicaraguaner, mit Ausnahme der in Artikel 49 des Statuts über die Rechte und Garantien erwähnten, ${ }^{26}$ außer Kraft gesetzt. Die Bestimmungen des Staatsnotstandes, durch die das Recht auf Gleichheit vor dem Gesetz, das Recht auf persönliche Freiheit und Sicherheit, die Rechte von Angeklagten und Verurteilten, die Rechtsweggarantien, das Recht des Habeas Corpus, das Recht auf Schutz vor willkürlichen Eingriffen ins Privatleben, die Unverletzlichkeit der Wohnung, das Brief- und Postgeheimnis, die Meinungs- und Informationsfreiheit, das Recht auf friedliche Versammlung und auf Teilnahme an Kundgebungen, die Vereinigungsfreiheit, das Recht auf Zusammenschluß zu politischen Zwecken, das Recht auf Eigentum, das Recht auf Arbeit, das Recht auf gewerkschaftlichen Zusammenschluß, das Streikrecht und noch weitere Rechte aufgehoben wurden, versetzten die Nicaraguaner in einen Zustand der Rechtlosigkeit. Auf der Grundlage dieses Gesetzes wurden die nachfolgend beschriebenen Antisomozistischen Volksgerichte eingerichtet, die Vorzensur für alle Kommunikationsmedien verhängt und die Ausstrahlung mehrerer Nachrichtenprogramme verboten.

Mitte 1984, während der Zeit vor den Wahlen, kam es zu einer leichten Milderung des Staatsnotstandes. ${ }^{27}$ Dabei wurden, jedenfalls formell, das Recht auf Teilnahme an öffentlichen Kundgebungen, die Vereinigungsfreiheit, das Recht auf Freizügigkeit in bestimmten, nicht zu militärischen Zonen erklärten Gebieten und das Streikrecht gewährt. ${ }^{28}$ Außerdem verpflichtete sich die Regierung dazu, die Vorzensur nur auf Nachrichten anzuwenden, die sich auf die Sicherheit des Staates beziehen. Das Habeas Corpus-Recht wurde nur den gewöhnlichen Gefangenen zugesprochen.

25 vgl. Art. 5 I, 2 . HS.

26 Recht auf Leben (Art. 5), Recht auf körperliche Unversehrtheit und Verbot der Folter (Art. 6), Verbot der Sklaverei (Art. 7), das Legalitätsprinzip (Art. 121), Gewissens- und Religionsfreiheit (Art. 19), Recht auf Teilnahme an öffentlichen Angelegenheiten, Petitionsrecht, aktives und passives Wahlrecht (Art. 25 b-d), das Recht auf Staatsangehörigkeit (Art. 26), Schutz der Familie (Art. 34), Rechte der Kinder (Art. 35).

27 Dekrete vom 19. Juli und 6. August 1984.

$28 \mathrm{Vgl}$. Amnesty International (Hrsg.) (Anm. 13), S. 7. 
Am 15. Oktober 1985 wurden diese "Zugeständnisse" neuerlich entzogen, und es wurde, was das Ausmaß der Einschränkungen anbelangt, der seit 1982 herrschende Zustand wiederhergestellt. Die Nationalversammlung nahm wenige Tage später einige Abänderungen am Staatsnotstand vor, die jedoch in der Praxis ohne Bedeutung blieben. ${ }^{29}$ Die nicaraguanische Rechtsanwaltskammer hat dieses am 15. Oktober 1985 verkündete Dekret über die Aufhebung von Rechten und Garantien als überflüssig und noch weitergehend als frühere entsprechende Maßnahmen verurteilt. ${ }^{30}$ Die unabhängige Menschenrechtskommission (CPDH) bewertete die Maßnahme der Regierung ebenfalls negativ im Hinblick auf eine friedliche Lösung der Probleme des Landes. ${ }^{31}$

Die Hoffnung, mit der Inkraftsetzung der neuen Verfassung würde der Staatsnotstand aufgehoben, erfüllte sich nicht. Nur wenige Stunden nach der Verkündigung am 9. Januar 1987 setzte Staatspräsident Daniel Ortega unter Berufung auf den Ausnahmezustand wichtige Grundrechtsartikel außer Kraft. Die Nationalversammlung billigte Mitte Februar 1987 mit ihrer sandinistischen Mehrheit dieses Dekret.

29 Den Abänderungen der Versammlung zufolge sollte die Zensur lediglich für militärische, wirtschaftliche oder die Staatssicherheit betreffende Angelegenheiten gelten. Die Einschränkung der Freizügigkeit sollte für Kriegsgebiete gelten und Versammlungen und Kundgebungen der vorherigen Genehmigung bedürfen. Das Habeas Corpus-Recht sollte nicht für Gefangene gelten, die des Verstoßes gegen das Gesetz über die öffentliche Ordnung beschuldigt wurden.

30 Vgl. Barra de Abogados de Nicaragua: Breve Historia del Estado de Suspensión de Derechos y Garantías de los Nicaragüenses bajo el Regimen del Gobierno Sandinista, Managua, 24. Oktober 1985; deutsche Ubersetzung in: Nicaragua-Gesellschaft e.V. (Hrsg.): Dokumente zur Menschenrechtslage, Nicaragua-Stimmen der Freiheit, Heft 4, Bonn 1986, S. 32 ff.

31 Comisión Permanente de Derechos Humanos: Nicaragua under the State of Emergency, San José, Februar 1986; tatsächlich setzte nach dem 15. Oktober 1985 eine neue Repressionswelle vor allem gegen die unabhängigen Gewerkschaften Central de Trabajadores de Nicaragua (CTN) und Confederación de Unificación Sindical (CUS), die politischen Oppositionsparteien, die katholische Kirche und die im Juni 1986 verbotene Zeitung La Prensa ein; vgl. dazu u. a. Interview mit CTN-Generalsekretär Carlos Huembes in: Informationsdienst Nicaragua (IDN), Ausgabe 23/85 vom 9. 11. 1985. Einen neuen Höhepunkt erreichten die Repressionsmaßnahmen im Juni/Juli 1986: Zu diesem Zeitpunkt wurden neben dem Verbot der La Prensa der Sprecher der katholischen Bischofskonferenz, Padre Bismarck Carballo, an der Wiedereinreise ins Land gehindert, der Bischof von Juigalpa, Msgr. Pablo Antonio Vega, wurde nach Honduras ausgewiesen. Die demokratischen Oppositionsparteien sind von massiven Verhaftungen betroffen. Nach Angaben der Parteivorsitzenden gegenüber den Verfassern befanden sich am Stichtag 12.7.1986 22 Mitglieder des PARTIDO SOCIAL CRISTIANO (PSC), 48 Mitglieder des PARTIDO LIBERAL INDEPENDIENTE (PLI), etwa 300 Mitglieder des PARTIDO CONSERVADOR DEMOCRATA DE NICARAGUA (PCDN), zumindest 1 Mitglied des PARTIDO SOCIAL DEMOCRATA (PSD) sowie nach Auskunft des Vorstandsmitglieds der CUS, Omar Vaca, fünf Gewerkschafter dieser Organisation in Haft. Lino Hernández, der Koordinator der CPDH, erklärte gegenüber den Verfassern, seit Dezember 1985 seien allein 2000 Festnahmen mit der Begründung erfolgt, die Beschuldigten arbeiteten mit den sog. Contras zusammen. Nach Angaben der CPDH gibt es in Nicaragua zur Zeit 6500 politische Gefangene, dazu noch etwa 2300 ehemalige Angehörige der Guardia Nacional, die seit 1979 in Haft sind. Innenminister Tomás Borge Martínez erklärte im Juli 1986, es gebe in Nicaragua etwa 2000 politische Gefangene bei einer Gefängnisbevölkerung von 8523 Personen, vgl. LA NACION vom 19. 7. 1986. 


\section{Antisomozistische Volksgerichte}

Durch das am 12. April 1983 im Gesetzblatt Nr. 82 veröffentlichte Dekret Nr. 1233 vom 11. April 1983 wurden von der Regierungsjunta des Nationalen Wiederaufbaus die sogenannten "Antisomozistischen Volksgerichte" (Tribunales Populares Antisomozistas TPA) eingesetzt. Die Aufgabe dieser "Rechtsprechungs"-Organe von zeitlich begrenztem Charakter bestand darin, "über die in den Artikeln 1 und 2 des Gesetzes über die Aufrechterhaltung der öffentlichen Ordnung und Sicherheit vorgesehenen Delikte" zu entscheiden. Sie sollten ihre Funktionen für die Dauer des Staatsnotstandes ausüben. Gemäß Art. 6 des Dekrets 1233 wird die gerichtliche Verhandlung über die Straftatbestände in dem in Dekret 896 verfügten summarischen Verfahren durchgeführt. Das heißt, es besteht eine Frist von zwei Tagen, um auf die Anklage zu antworten, eine Frist von acht Tagen (verlängerbar auf zwölf) für die Beweisaufnahme und eine Frist von drei Tagen bis zur Verkündung des Urteils. Die Möglichkeit, bei einem ordentlichen Gericht das Rechtsmittel der Berufung oder beim Obersten Gerichtshof das Rechtsmittel der Revision einzulegen, bestand hierbei jedoch nicht. Berufung konnte nur beim Antisomozistischen Volksgericht zweiter Instanz eingelegt werden.

Kein Aspekt des revolutionären nicaraguanischen Rechtssystems hat zu größeren Kontroversen geführt als die Errichtung dieser "außergerichtlichen" Gerichte mit dem Ziel, politisch sensible Fälle abzuurteilen..$^{32}$ Nach Auffassung des Lawyers Committee for International Human Rights hat dieses Experiment der Regierung mit der "Volksgerichtsbarkeit" das reguläre Gerichtswesen unterlaufen und für die Bürger die Ausübung der Rechte beschnitten, die durch Gerichte garantiert werden sollen. Die unabhängige nicaraguanische Menschenrechtskommission $\mathrm{CPDH}$ rügt in ihrem umfassenden Bericht über die Verletzung der Menschenrechte durch gesetzliche Bestimmungen der Revolutionsregierung die Schaffung dieser Gerichte als Verstoß gegen das Prinzip der Gleichheit vor dem Gesetz sowie gegen den Anspruch auf ein unparteiisches Gerichtsverfahren, wie es in von Nicaragua ratifizierten internationalen Menschenrechtskonvention garantiert wird. Die Kommission qualifiziert die Antisomozistischen Volkstribunale als eine "neue, noch bösartigere Spielart" der durch das Dekret 643 vom 3. Februar 1983 aufgelösten Sondergerichte. ${ }^{33}$

Amnesty International äußert sich in seinem Bericht vom März $1986^{34}$ zum Charakter dieser Volksgerichte folgendermaßen:

"Von weiterer Bedeutung für das Recht auf ein faires Gerichtsverfahren durch ein unabhängiges Gericht ist die Tatsache, daß das TPA-System aus dem ordentlichen Gerichtssystem und aus dem Geltungsbereich der Uberwachung durch den obersten Gerichtshof fällt. Kein höheres Gericht kann Urteile überprüfen oder das Verhalten der Richter überwachen. Anders als Urteile, die von ordentlichen Gerichten gefällt

32 Lawyers Committee for International Human Rights (Anm. 5), S. 33.

33 Vgl. Nicaragua-Gesellschaft e.V. (Hrsg.) (Anm. 7), S. 30.

34 Amnesty International (Hrsg.) (Anm. 13): S. 21. 
werden und gegen die Rechtsmittel beim Obersten Gerichtshof eingelegt werden können, können gegen Urteile, die von den Volkstribunalen gefällt werden, keine Rechtsmittel außerhalb des TPA-Systems eingelegt werden. (Zur Zeit besteht das TPA-System aus einem Vorinstanzgericht und einem Berufungsgericht, die sich beide in der Hauptstadt Managua befinden). Die Mitglieder der Gerichte werden auf unbestimmte Zeit durch die Exekutive ernannt und zu jeder Kammer gehört ein Anwalt - in der Praxis aus dem Personal des Justizministeriums ausgewählt - und zwei andere, die von den der Frente Sandinista de Liberación Nacional (FSLN) angegliederten Organisationen benannt werden. Im Gegensatz dazu werden die Richter der ordentlichen Gerichte durch den Obersten Gerichtshof ernannt."

Die private Menschenrechtsorganisation America's Watch äußerte sich schon vorher ähnlich. Sie hat die Befürchtung, daß nicht unterschieden wird zwischen Verbrechen gegen den Staat einerseits und Verbrechen gegen den Sandinismus andererseits. ${ }^{35}$ Nach Ansicht von America's Watch sollten diese Sondergerichte abgeschafft werden, zumal im Jahre 1985 die Verurteilungsrate außerordentlich hoch war, und die Urteile sehr hart ausfielen. ${ }^{36}$

Obwohl Vertreter des Regimes, wie Innenminister Tomás Borge oder der ehemalige Präsident des Obersten Gerichtshofs, Roberto Argüello, die Notwendigkeit der neuen Antisomozistischen Volksgerichte mit dem Bedürfnis nach schneller und effektiver Justizgewährung der Angeklagten begründeten, verbringen diese oftmals lange Monate in Haft, bevor sie abgeurteilt werden. ${ }^{37}$ Zwar wird in dem Gesetz über die Einrichtung der TPAs verfügt, daß diese Gerichte nur über Delikte urteilen sollen, die einen Verstoß gegen das Gesetz über die öffentliche Ordnung und Sicherheit darstellen, doch gibt es Äußerungen von Funktionären der Regierung, die darauf gerichtet sind, diese "Rechtsprechung" auszuweiten.

Im Gegensatz zu Argüello erklärte der Justizminister seinerzeit, daß diese Gerichte nicht über diejenigen "Contras" Recht sprechen sollten, die im Kampfe gefangengenommen werden würden, sondern vielmehr über diejenigen, die im Innern des Landes aktiv wären und dort Tätigkeiten wie die der Spekulation, der Sabotage an Produktion und Wirtschaft und der Verbreitung von "reaktionären Gerüchten" ausübten. ${ }^{38}$

Innenminister Borge ging noch einen Schritt weiter, als er kritisierte, daß die "Gesetze des Somozismus" in Kraft blieben und die Richter an den ordentlichen Gerichten nur mechanisch die Gesetze anwendeten, "ohne die Bedingungen, unter denen wir leben, in Betracht zu ziehen" und damit Resultate ermöglichten, die teilweise im Widerspruch zu

35 America's Watch: Human Rights in Nicaragua, (The Special Courts), New York/Washington, April 1984.

36 Vgl. dazu im Einzelnen America's Watch: Human Rights in Nicaragua, 1985-1986, New York/Washington, March 1986, S. 44; nach Kriele kamen auf 910 Anklagen vor den TPAs 26 Freisprüche, bei denen es sich auch noch um "agents provocateurs" gehandelt haben soll; vgl. Kriele, Martin: Nicaragua - das blutende Herz Amerikas. Ein Bericht, 4. Aufl., München/Zürich 1986, S. 62.

$37 \mathrm{Vgl}$. Lawyers Committee for International Human Rights (Anm. 5), S. $43 \mathrm{f}$.

38 Vgl. die Äußerungen von Roberto Arguello in: Lawyers Committee for International Human Rights (Anm. 5), S. 43 einerseits und die Meldung in BARRICADA vom 23. Mai 1983 andererseits. 
den Prinzipien der revolutionären Justiz stünden. ${ }^{39}$ Nach Auffassung des Innenministers bieten die Antisomozistischen Volksgerichte die Mittel, um die "Prinzipien der Revolution" durchzusetzen, denn ihre Mitglieder wendeten nicht in strenger Weise die Regeln des Beweisrechts an, wenn sie glaubten, daß diese Regeln falsche Ergebnisse produzieren würden.

In Äußerungen solcher Art kommt der eigentliche Charakter dieser Sondergerichte zum Vorschein. Aufgrund der wiederholten Verlängerung des Staatsnotstandes üben diese Gerichte nach wie vor ihre Funktionen aus und gehören zu den wichtigsten Repressionsinstrumenten des revolutionären Staates gegen kritische Bürger. ${ }^{39 a}$

\section{Gesetze über die Kommunikationsmedien}

Der Schutz der Meinungsfreiheit wurde in Artikel 8 des Grundsatzstatuts verbürgt, in dem es hieß:

"Die Gewissens- und Religionsfreiheit, begründet in dem umfassendsten Geist der Toleranz, die uneingeschränkte Freiheit der Meinungsäußerung in Wort oder Schrift und das Recht, sich politisch oder gewerkschaftlich zu organisieren, werden anerkannt, solange sie nicht aus dem Statut über die Rechte und Garantien der Nicaraguaner hervorgehenden Einschränkungen unterworfen sind."

Solche Einschränkungen ergaben sich aus Art. 20 und Art. 21 des Status über die Rechte und Garantien der Nicaraguaner. In Art. 20 hieß es:

"Die Informationsfreiheit ist eines der wesentlichen Prinzipien einer echten Demokratie und kann deshalb, weder direkt noch indirekt, der wirtschaftlichen Macht irgendeiner Gruppe unterstellt werden".

Artikel 21 des Status lautet:

"Jedermann hat das Recht auf Gedankenfreiheit und auf Meinungsäußerung; dieses Recht schließt die Freiheit ein, sich Informationen und Gedankengut jeder Art in Wort, Schrift oder Druck, durch Kunstwerke oder andere Mittel eigener Wahl zu beschaffen, zu empfangen und weiterzugeben. Die Ausübung dieser Rechte umfaßt Pflichten und Verantwortung und kann daher bestimmten Formalitäten, Bedingungen oder Einschränkungen unterzogen werden, die das Gesetz festlegt, und die erforderlich sind:

a) im Interesse der staatlichen Sicherheit und Integrität, der öffentlichen Sicherheit und der nationalen Wirtschaft;

b) der Verteidigung der Ordnung und der Verhütung von Verbrechen;

39 Interview mit Innenminster Tomás Borge vom 14. Juni 1984 in: Lawyers Committee for International Human Rights (Anm. 5), S. 46 f.

39a Nach Aussagen des Nationalen Koordinators der COMISION PERMANENTE DE DERECHOS HUMANOS (CPDH), Lino Hernández, gegenüber den Verfassern wareteten im Juli 19862500 Häftlinge auf ihren Prozeß vor den TPAs; vgl. Rheinischer Merkur vom 29. 8. 1986, S. 28. 
c) zum Schutz der Gesundheit und der Moral, der Würde der Menschen und des Rufs und der Rechte anderer;

d) zur Vermeidung der Verbreitung von vertraulichen Informationen oder zur Gewährleistung der Autorität und Unparteilichkeit der Gerichte".

Artikel 20 des Status über die Rechte und Garantien enthielt nicht nur verschiedene Einschränkungen hinsichtlich der Ausübung der Meinungsfreiheit, sondern es fehlten auch einige der Garantien für die Ausübung dieses Rechts, die in verschiedenen internationalen Konventionen verbürgt sind. Dazu zählte das Verbot der Vorzensur und des Mißbrauchs staatlicher Kontrollen in Form indirekter Maßnahmen. ${ }^{40}$

Eine erste Bestimmung, die das Recht auf freie Meinungsäußerung beschnitt, fand sich bereits in Artikel 4, Absatz c, des Gesetzes zur Aufrechterhaltung der öffentlichen Ordnung und Sicherheit. Danach konnten Personen zu Zwangsarbeit von 3 Monaten bis zu 2 Jahren verurteilt werden, die das folgende Delikt begangen hatten:

"c) die Verbreitung durch Wort oder Schrift von Äußerungen, Bekanntmachungen oder Erklärungen, mit denen gegen das Gemeinwohl verstoßen und beabsichtigt wird, die Erfolge und Errungenschaften des Volkes zunichte zu machen und seine Interessen zu verletzen".

Diese vage und unpräzise Definition ermöglicht es, jede Abweichung von der Ideologie und Politik der Regierung oder des betreffenden Richters, sowie jede Kritik an der Politik der jeweils regierenden Gruppe zu bestrafen.

Dekret Nr. 48 vom 16. August 1979 (Das allgemeine Gesetz über gesellschaftliche Medien) vermittelt in seinem Artikel 2, Absatz b, ein deutliches Bild von dem frühen Versuch der sandinistischen Regierung, die Informationsfreiheit einzuschränken. Dort heißt es hinsichtlich der Kritik an Staatsbeamten und jeder Art von "Informationsarbeit":

"b) die im vorigen Absatz beschiebenen Veröffentlichungen müssen ein echtes Engagement für die Verteidigung der Errungenschaften der Revolution, für den Prozeß des Wiederaufbaus und für die Probleme des nicaraguanischen Volkes zum Ausdruck bringen und nicht zu Instrumenten volksfeindlicher Interessen werden".

Das Gesetz bestimmte auch die Bildung eines Komitees, bestehend aus dem Kulturminister, einem Vertreter der Kommunikationsmedien und einem Vertreter des Verbandes nicaraguanischer Journalisten, ${ }^{41}$ das die Begriffe des Gesetzes interpretieren und über die Verstöße gegen dieses Gesetz entscheiden soll. In der Praxis übte dieses Komitce niemals seine Funktion aus, und derjenige, der eigentlich diese Tätigkeiten ausübte, war der Koordinator der Abteilung für Kommunikationsmedien, der später dem Innenministerium zugeordnet wurde. Unter anderem gehört es zu den Aufgaben dieses Koordinators:

- die Verkettung aller Radio- und Fernsehstationen zu bewirken, wenn "aus Gründen der Staatsräson" die Verbreitung von Nachrichten oder die Úbertragung von Radiound Fernsehauftritten von transzendentaler Bedeutung für das nicaraguanische Volk sind,

40 Vgl. dazu die Auffassung der COMISION PERMANENTE DE DERECHOS HUMANOS (PDH) in: Nicaragua-Gesellschaft e.V. (Hrsg.) (Anm. 7): S. 17 ff.; America' Watch (Anm. 36): S. 47.

41 Dabei handelt es sich um die pro-sandinistische UNION DE PERIODISTAS NICARAGUENSES (UPN). 
- die Erlaubnis für die direkte Úbertragung von ausländischen Programmen zu erteilen,

- dafür zu sorgen, daß Ubertragungen nicht gegen die im Gesetz über die Medien enthaltenen Bestimmungen verstoßen.

Das Dekret Nr. 48 sieht darüber hinaus eine ausgesprochen scharfe Kontrolle der elektronischen Kommunikationsmedien vor, die vom Amt für Genehmigungen und Zulassungen ausgeübt werden soll. Zu den Befugnissen dieses Amtes gehört es, Konzessionen zu erteilen oder zu entziehen sowie deren Nichtigkeit oder Erlöschen zu verfügen, die Arbeit der Radiostationen und deren Dienstleistungen zu überwachen und in jedes Handelsgeschäft einzugreifen, das gegen die Eigentumsordnung dieser Medien verstößt. ${ }^{42}$ Konzessionen können wieder entzogen werden, wenn deren Inhaber seine nicaraguanische Staatsangehörigkeit aufgibt oder wenn er eine ausländische Regierung, ein ausländisches Unternehmen oder eine ausländische Person um Ubernahme der Schirmherrschaft ersucht. ${ }^{43}$ All diese Entscheidungen können gefällt werden, ohne daß man dem Betroffenen das Recht auf Verteidigung gewährt und ohne daß sein Fall von einem der dafür zuständigen Gerichte entschieden wird.

Artikel 37 der Verordnung legt Rundfunk, Fernsehen und Presse auf Konformität mit dem offiziellen Denken fest. Es heißt dort, daß mihre Tätigkeit vornehmlich darauf ausgerichtet sein muß, einen Beitrag zur Sicherung unserer revolutionären Errungenschaften und zur Verteidigung unserer Revolution zu leisten...."

Fast unbemerkt wurden von der Regierung die Entscheidungsbefugnis sowie alle Aufgaben und Zuständigkeiten, die den Tätigkeitsbereich der Massenmedien betreffen, vom Kultusministerium auf das Innenministerium übertragen. Durch die Verlagerung der Kontrolle über die gesprochenen und gedruckten Medien ins Innenministerium wurden die Einschränkungen des Rechts auf freie Meinungsäußerung, die das Gesetz den Medien auferlegt, in der Praxis weiter verschärft.

Andere Dekrete, die die Meinungsfreiheit einschränken, sind die Dekrete 511 und 512 vom 10. September 1980, die die Veröffentlichung von Informationen über die innere Sicherheit und wirtschaftliche Themen betreffen. Den Kommunikationsmedien ist es danach untersagt, Nachrichten, die eine Gefahr für oder einen Angriff auf die innere Sicherheit des Landes und die Landesverteidigung darstellten, sowie Informationen wirtschaftlichen Inhaltes zu verbreiten.

Verstöße gegen die betreffenden Dekrete sollten gemäß den Bestimmungen des Gesetzes über die Aufrechterhaltung der öffentlichen Ordnung und Sicherheit strafrechtlich verfolgt werden. Auch hierbei wurde die Vorzensur legalisiert, da die in Frage kommenden Informationen zunächst der Regierungsjunta und den Ministerien des Innern, der Verteidigung und des Binnenhandels vorgelegt werden sollten. Per Dekret 707 vom 25. April 1981 wurde dann später der Text der vorausgegangenen Dekrete 511 und 512 in das all-

42 Art. 4 und 5 der Ausführungsverordnung zum Gesetz über die Medien, vgl. LA GAZETA vom 19. September 1979.

43 Art. 17 Abs. 5 der Ausführungsverordnung. 
gemeine Gesetz über die Kommunikationsmedien aufgenommen. Bei Nicht-Einhaltung der betreffenden Verfügung sollten die Medien mit dem vorübergehenden oder vollständigen Verbot ihrer Sendungen oder Publikationen bestraft werden.

Für die Anwendung all dieser auf das Gesetz über die Kommunikationsmedien bezogenen Vorschriften hat jedoch seit März 1982 kein Anlaß bestanden. Seit dieser Zeit ist der Staatsnotstand in Kraft, der von seinen Auswirkungen her für die sandinistischen Behörden effektiver ist, da unter seiner Geltung alle Kommunikationsmedien der Vorzensur unterworfen sind. In dem betreffenden Zeitraum wurden mehr als 20 Nachrichtenprogramme definitiv eingestellt. ${ }^{44}$

\section{Gesetz über die politischen Parteien und Wahlgesetz}

Artikel 25 des Statuts über die Rechte und Garantien der Nicaraguaner legte fest, welches die politischen Rechte sind:

"Alle Bürger genießen uneingeschränkt folgende Rechte:

a) Parteien oder politische Gruppen zu organisieren oder ihnen beizutreten;

b) an der Führung öffentlicher Angelegenheiten unmittelbar oder durch frei gewählte Vertreter mitzuwirken;

c) selbständig oder gemeinsam mit anderen schriftliche Petitionen an Staatsbeamte, Behörden oder die Staatsgewalt zu richten, sowie das Recht auf baldigen Bescheid;

d) aktives und passives Wahlrecht, Zulassung zu öffentlichen Ämtern unter gleichen Bedingungen."

Per Dekret 1312 vom 2. September 1983 wurde das Gesetz über die politischen Parteien verkündet. Artikel 5 dieses Gesetzes enthält die Verpflichtung der politischen Parteien, "... die Grundprinzipien der sandinistischen Volksrevolution zu respektieren, als da sind der Anti-Imperialismus sowie ihre Eigenschaft als eine tief im Volk verwurzelte und demokratische Bewegung". Artikel 7 des Gesetzes verpflichtet die politischen Parteien darauf, "die Revolution gegen jeden von innen oder außen kommenden Versuch zu verteidigen, in Nicaragua ein Regime der Unterdrückung und Ausbeutung einzuführen". Ein Verstoß gegen diese Bestimmungen wird mit dem vorübergehenden Verbot der Partei bestraft. Im Wiederholungsfalle muß die Partei mit einem vollständigen Verbot rechnen.

44 Vgl. dazu Buss, Hero, Pressef reiheit in Nicaragua, in: Nicaragua-Gesellschaft e.V. (Hrsg.): Beiträge zur Sache, Nicaragua-Stimmen der Freiheit, Heft 3, Bonn 1986, S. 35 ff.

Zuletzt wurde am 1. Januar 1986 das staatsunabhängige Radioprogramm, RADIO CATOLICA verboten, da sich dessen Direktor zunächst geweigert hatte, die Neujahrsansprache von Staatspräsident Daniel Ortega Saavedra zu übertragen. Die einzige regierungskritische Tageszeitung, LA PRENSA, die mit bis zu 70000 verkauften Exemplaren größte des Landes, wurde am 26. Juni 1986 auf Anordnung der Mediendirektion des Innenministeriums auf unbestimmte Zeit geschlossen 
Diese Bestimmungen erlegen allen Parteien die Verpflichtung auf, die Symbole, Prinzipien und Praktiken des Frente Sandinista de Liberación Nacionál (FSLN) und seiner Organisationen zu respektieren, denn gemäß Dekret Nr. 67 vom 13. September 1979 bleibt die Bezeichnung "sandinistisch" dem FSLN vorbehalten. Wenn also von der sandinistischen Revolution gesprochen wird, dann ist unter Revolution die im Sinne der Sandinistischen Front praktizierte Revolution zu verstehen. Konkret bedeutet das die Verpflichtung, die Prinzipien zu verteidigen, die von der Partei an der Macht vorgegeben werden.

Eine der im Gesetz über die politischen Parteien enthaltenen Vorschriften zur politischen Propaganda und Überzeugungsarbeit wird in der Ausführungsverordnung zu diesem Gesetz $^{45}$ wieder aufgegriffen. In Artikel 9 der Ausführungsverordnung heißt es:

"In Úbereinstimmung mit den geltenden Gesetzen kann eine ständige Propaganda über die folgenden Medien verbreitet werden: . . über Presse, Rundfunk und Fernsehen, den wirtschaftlichen Möglichkeiten jeder Partei entsprechend und unter Wahrung des Rechts der freien unternehmerischen Tätigkeit."

Der letzte Teil des Abschnitts birgt eine Tücke in sich, die darin besteht, daß die politische Propaganda vom Recht der freien unternehmerischen Tätigkeit abhängig gemacht wird. Dazu muß man wissen, daß zum damaligen Zeitpunkt bereits über $85 \%$ der Radiostationen in Nicaragua im Besitz der Regierung oder von prosandinistischen Kooperativen waren, daß weiterhin sämtliche Fernsehkanäle der Regierung gehörten und daß von den ehemals drei im Lande erscheinenden Zeitungen nur eine unabhängig und der Vorzensur unterworfen war.

Nach dem Verbot von La Prensa am 26. Juni 1986 erscheinen nur noch das Parteiblatt des FSLN, Barricada, und die regierungsnahe Tageszeitung El Nuevo Diario.

Derselbe Abschnitt verfügt unter anderem auch, daß in der politischen Werbung gegenüber der sandinistischen Volksrevolution ein "besonderer Respekt" gewahrt werden muß. Verstölße gegen diese Verfügung werden mit Arrest bestraft. Wie aus dem Text des Gesetzes selbst hervorgeht, besteht für die übrigen Parteien im Verhältnis zur sandinistischen Partei weder eine Gleichheit der politischen Rechte noch eine Gleichheit vor dem Gesetz.

Noch konkretere Gefahren drohen oppositionellen Parteien in dem durch Art. 3 geforderten Anerkennungsverfahren. Nur anerkannte Gruppen genießen nach dieser Bestimmung die Rechte und Garantien des Gesetzes. Das wichtigste Organ in diesem Prozeß (Art. 19 ff.) und in den Fällen der Suspendierung (Art. 26, 27) und Auflösung (Art. 28) ist der Nationalrat der Politischen Parteien (CNPP), dessen Kompetenzen gemäß Art. 16 nahezu umfassend sind.

Der Nationalrat entscheidet über die Nichterfüllung der in Art. 7 genannten Pflichten der Parteien. Die Nichterfüllung dieser Pflichten stellt im Wiederholungsfall einen Auflösungsgrund dar (Art. 28a). Bedenklich erscheint auch die Möglichkeit, eine Partei gem. Art. 27a zu suspendieren, wenn sie sich aus dem Staatsrat zurückzieht. Eine einmal 
aufgelöste politische Partei darf nicht wieder unter dem gleichen Namen und Emblem oder mit dem gleichen Parteivorstand gegründet werden (Art. 33). Die Machtfülle des CNPP ist umso bedenklicher, wenn man einen Blick auf seine Zusammensetzung wirft. Nach Art. 15 gehören dem Nationalrat der Politischen Parteien vier Delegierte aus der nationalen Versammlung der Politischen Parteien (ANPP) an, die nach Art. 10 aus Vertretern aller Parteien besteht. Dazu kommen der Vorsitzende des CNPP, der von der Regierungsjunta entsandt wird, und drei vom Staatsrat benannte Mitglieder. ${ }^{45 \mathrm{a}}$ Das Wahlgesetz wurde per Dekret 1413 vom 26. März 1984 verkündet. ${ }^{46}$ Gemäß seinem Artikel 2 "hat dieses Gesetz zum Ziel, den Wahlvorgang, der verstanden wird als die Gesamtheit der auf die Wahl der ausführenden und gesetzgebenden Staatsgewalten ausgerichteten Aktivitäten, in all seinen Einzelheiten und in Ubereinstimmung mit dem Grundsatzstatut und seiner Reform zu regeln" ${ }^{47}$

In Artikel 3 des Dekrets werden die für die Wahl zuständigen Stellen bestimmt, die mit der Anwendung des Gesetzes beauftragt sind:

- der Oberste Wahlrat,

- die auf regionaler Ebene tätigen Wahlräte,

- die auf Bezirksebene tätigen Wahlausschüsse,

- die für die Annahme der Wahlstimmen zuständigen Ausschüsse.

Der Oberste Wahlrat setzt sich zusammen aus fünf Mitgliedern, die vom Obersten Gerichtshof ernannt werden. Gegen die Beschlüsse, die von dieser Stelle getroffen werden, kann kein ordentliches oder außerordentliches Rechtsmittel eingelegt werden.

Die Besetzung der übrigen für die Wahl zuständigen Stellen obliegt dem Obersten Wahlrat, wobei den anderen politischen Parteien nicht einmal die Möglichkeit eingeräumt wird, in irgendeiner Weise an der personellen Zusammensetzung dieser Stellen mitzuwirken.

Das Gesetz enthält Bestimmungen, die sich auf den Wahlkampf und die Wahlpropaganda im gesamten Bereich der Massenmedien beziehen und regelt die Nutzung der für die Politik zur Verfügung stehenden Sendezeiten in Rundfunk und Fernsehen. Danach hat jede Partei Anspruch auf dreizehn Minuten Sendezeit pro Woche im Fernsehen und auf dreieinhalb Minuten Sendezeit täglich im Radio. Eine solche Zuteilung von Zeit für die Durchführung von Wahlpropaganda muß als unzureichend betrachtet werden, wenn man davon ausgeht, daß die Regierungspartei bereits während der den Wahlen am 4. 11. 1984 vorausgegangenen vier Jahre fortlaufend über alle Kommunikationsmedien des

45a Kritisch zum Parteiengesetz Informationsdienst Nicaragua (IDN) 12/83, 15. 10. 1983 (Nicaraguanisches Parteiengesetz - Skepsis angebracht, S. 2 f.); vgl. dazu ausführlich auch Wolf (Anm. 4): S. $180 \mathrm{ff}$.

46 In deutscher Úbersetzung abgedruckt bei: Koch, Jürgen: Wahlen in Nicaragua. Dokumentation. München 1984, S. $88 \mathrm{ff}$.

47 Per Dekret 1399 vom 21. Februar 1984 wurde das Grundsatzstatut abgeändert. Gemäß Artikel 28 war zuvor, bei Ausschreibung durch die Regierungsjunta, die Durchführung von allgemeinen Wahlen zur Bildung einer Nationalversammlung vorgesehen. Nach der Änderung sollten Wahlen ausgeschrieben werden für die Ernennung des Präsidenten, des Vizepräsidenten und zur Errichtung der Nationalversammlung mit verfassungsgebenden und gesetzgeberischen Kompetenzen. 
Staates politische Kampagnen durchführen konnte, ohne daß die Zensur oder das Notstandsgesetz sie daran gehindert hätte.

Das Gesetz verfügt weiterhin ein Verbot von Propaganda zum Zwecke des Aufrufs zur Wahlenthaltung und garantiert außerdem das Recht auf allgemeine und geheime Wahlen. Alle Personen ab 16 Jahren sind wahlberechtigt. Den Militärs wird ebenfalls die Wahlberechtigung erteilt. Der erforderliche Nachweis der Identität kann durch Vorlage der Geburtsurkunde, der Sozialversicherungskarte, des Führerscheins, des Reisepasses oder durch "jedes andere Dokument, das der Identifikation dienen kann", erbracht werden.

Diese Bestimmungen führten zu heftigen Auseinandersetzungen, u. a. weil das Gesetz über die Staatsangehörigkeit abgeändert werden mußte, um die Teilnahme der Jugendlichen ab 16 an der Wahl zu ermöglichen. Außerdem stellte die Erteilung der Wahlberechtigung an die Militärs eine Neuigkeit dar, denn in allen vorausgegangenen Gesetzestexten war die Teilnahme der Militärs an den Wahlen verboten.

Artikel 27 des Wahlgesetzes im Zusammenhang mit dem Gesetz über die politischen Parteien sieht vor, daß nur diejenigen Parteien als politische Parteien mit Rechtsfähigkeit anerkannt werden, die sich, allein oder in Bündnissen, zur Wahl anmelden, an den Wahlen teilnehmen und von denen mindestens ein Kandidat als Vertreter in die Nationalversammlung gewählt wird.

Diejenigen politischen Parteien, die ihre Rechtsfähigkeit verlieren, können weiterhin als politische Gruppierung tätig sein. Diese Bestimmung wird von der demokratischen Opposition als Angriff auf den politischen Pluralismus betrachtet. Er setzte die politischen Parteien unter Druck, um sie zur Teilnahme an den Wahlen zu bewegen. Denjenigen, die das nicht taten, blieb nichts anderes übrig, als sich in die politische Verbannung zu begeben. ${ }^{48}$

Das Wahlgesetz weist zwar die beschriebenen schwerwiegenden Mängel auf, jedoch bestand 1984 das Hauptproblem darin, daß ein für die Durchführung von Wahlen geeignetes politisches Klima fehlte. Das lag daran, daß die Wahlen unter den Bedingungen des Staatsnotstands abgehalten wurden und daß außerdem eine Reihe von Gesetzen in Kraft war, durch die die individuellen und kollektiven Freiheiten eingeschränkt wurden. ${ }^{49}$

48 Im August 1984, nachdem die in der COORDINADORA DEMOCRATICA NICARAGUENSE (CDN) zusammengeschlossenen Oppositionsparteien ihren Beschluß über die Nichtteilnahme an den Wahlen bekanntgegeben hatten, wurde vom Nationalrat der Politischen Parteien die Aufhebung der Rechtspersönlichkeit des PARTIDO SOCIAL CRISTIANO (PSC), des PARTIDO SOCIAL DEMOCRATA (PSD) und des PARTIDO LIBERAL CONSTITUCIONALISTA (PLC) beschlossen; vgl. dazu das Interview mit dem damaligen Präsidenten des PSC, Agustín Jarquín Anaya, in: Nicaragua-Gesellschaft e.V. (Hrsg.): Gespräche mit Agustín Jarquín Anaya, Edén Pastora Gómez, Indalecio Rodríguez Alaniz und Brooklyn Rivera, Nicaragua-Stimmen der Freiheit, Heft 1, Bonn 1984, S. 9 ff.; das Interview mit dem Spitzenkandidaten des Oppositionsbündnisses der COORDINADORA DEMOCRATICA NICARAGUENSE (CDN), Arturo Cruz Porras, in: Lateinamerika Report Nr. 10/1984, S. 34 ff.; das Interview mit dem damaligen Präsidenten der CDN, dem Generalsekretär des PARTIDO SOCIAL DEMOCRATA (PSD), Luis Rivas Leiva, in: Koch, Jürgen (Anm. 46): S. 73 ff.; Velazquez, José Luis: Sociedad Civil y Dictadura, San José 1986, S. 122.

49 Nach amtlichen Angaben sollen am 4. 11. 1984 bei der Präsidentschaftswahl insgesamt 1170162 Stimmen abgegeben worden sein, was einer Wahlbeteiligung von $75,42 \%$ entspricht. Daniel Ortega Saavedra und Ser- 


\section{Die neue Verfassung Nicaraguas}

Mit den Wahlen zur Verfassungsgebenden Versammlung am 4. November 1984 löste bei aller berechtigter Kritik - die Regierungsjunta eine wesentliche Verpflichtung des Grundsatzstatuts ein. Sie schuf damit zugleich die Voraussetzungen für den etwa zwei Jahre dauernden Verfassungsprozeß. Die in der COORDINADORA DEMOCRATICA NICARAGÜENSE zusammengeschlossenen demokratischen Oppositionsparteien sind, da sie an den Wahlen nicht teilnahmen, nicht in der Nationalversammlung (Asamblea Nacional) vertreten, was für die Verfassungsdiskussion von nicht unerheblicher Bedeutung war. ${ }^{50}$

Der Auftrag zur Ausarbeitung und Bestätigung der Verfassung findet sich in Art. 24 Nr. 1 des am 20. März 1985 verabschiedeten Allgemeinen Statuts für die Nationalversammlung (Gesetz Nr. 3). Am 21. Mai 1985 gründete die Nationalversammlung die "Comisión Especial Constitucional“ (CEC). Vorsitzender wurde in Personalunion der Parlamentspräsident, Carlos Nuñez Tellez. Ihr oblag die organisatorische Durchführung des Verfassungsprozesses. ${ }^{51}$ Mit der Verhängung des verschärften Ausnahmezustandes am 15. Oktober 1985 verhinderte die Regierung selbst eine breite politische Beteiligung an der Ausarbeitung der neuen Verfassung. Nicht nur die außerparlamentarische demokratische Opposition, sondern auch die im Parlament vertretene liberale Partei PLI und die sozialistische Partei PSN kündigten ihre Mitarbeit auf, da es unter den Umständen des Staatsnotstandes absurd sei, über die Verfassung zu diskutieren. ${ }^{52}$ Damit war der letzte

gio Ramírez Mercado, die vom FSLN als Kandidaten für die Ämter des Staatspräsidenten und des Vize-Präsidenten benannt worden waren, erhielten danach 66,9\% der Stimmen. Bei der gleichzeitigenWahl der Abgeordneten der verfassungsgebenden Nationalversammlung wurden nach amtlichen Angaben 1170102 Stimmen abgegeben. In der verfassunggebenden Nationalversammlung, die 96 Abgeordnete umfaßt und sechs Jahre lang tätig sein wird, gingen 61 Sitze an den FRENTE SANDINISTA DE LIBERACION NACIONAL (FSLN), 9 an den PARTIDO LIBERAL INDEPENDIENTE (PLI), 6 an den PARTIDO POPULAR SOCIAL CRISTIANO (PPSC) und je 2 an den PARTIDO SOCIALISTA NICARAGUENSE (PSN), den PARTIDO COMUNISTA DE NICARAGUA (PCdeN) und das MOVIMIENTO DE ACCION POPU. LAR/MARXISTA-LENINISTA (MAP/ML). Zur weitverbreiteten Kritik an diesen Wahlen vgl. statt vieler: Gerhard Elschner: Leider nur ein Täuschungsmanöver. Manipulierte Wahlen in Nicaragua, in: J. Koch (Anm. 46), S. 26 ff.; Kriele (Anm. 35): S. 52 ff.; Informationsdienst Nicaragua (IDN): 24/84 vom 24.11. 1984.

50 Einige Parteien und politische Gruppen haben aber schriftliche Veröffentlichungen zur Verfassungsdiskussion vorgelegt. So z. B. : PARTIDO CONSERVADOR DE NICARAGUA (PCN): Analisis del proyecto sandinista de constitución politica, Managua, März 1986; PARTIDO CONSERVADOR DEMOCRATA DE NICARAGUA (PCDN), Clemente Guido: Consideraciones sobre el proyecto de constitución politica de Nicaragua, Managua, Juni 1986; die Exilgruppe UNIDAD NICARAGUENSE OPOSITORA (UNO), Mario Benito Darce: Proyecto sandinista de constitución. 20 puntos basicos para la construcción de una democracia popular, San José, Februar 1986. Der PARTIDO LIBERAL INDEPENDIENTE (PLI) und der PARTIDO SOCIAL CRISTIANO (PSC) haben Alternativentwürfe ausgearbeitet. Im April 1986 hatte der PCDN einen 17 Punkte umfassenden Änderungsantrag über den Präsidenten der Nationalversammlung, Comandante Carlos Nuñez, eingebracht, über den jedoch nicht verhandelt wurde, vgl. dazu Informationsdienst Nicaragua (IDN), Ausgabe 13/86 vom 21. 5. 1986.

51 Eingehend dazu Böhler in KAS-Auslands-Informationen 9/86, S. 3 ff., St. Augustin 1986.

52 LA PRENSA vom 19. 11. 1985 (zensiert), zit. nach Böhler (Anm. 51), S. 5 mit weiteren Nachweisen; vgl. auch INFORMATIONSDIENST NICARAGUA (IDN) 18/86 v. 30. 8. 1986, S. 1 f. (Interview mit Virgilio Godoy, PLI). 
Rest an gemeinsamen, pluralistisch orientierten Überlegungen verloren. Die sandinistische Regierung war nunmehr in der Lage, ungehindert ihr Konzept einer staatlichen Ordnung umzusetzen.

Daran änderten auch nichts die sogenannten "Cabildas Abiertas", öffentliche Volksversammlungen, die von der Regierung durchgeführt wurden, um die Bevölkerung an der Willensbildung zu "beteiligen". Dort beteiligten sich im wesentlichen Mitglieder und Aktivisten des FSLN und ihm nahestehende Massenorganisationen,,$^{53}$ und Kritik äußerte sich allenfalls an zweitrangigen Fragen.

Ohne hier eine ins Detail gehende Exegese vornehmen zu wollen, soll nachfolgend exemplarisch gezeigt werden, in welchem Verhältnis die neue Verfassung zum ursprünglichen Regierungsprogramm und den Zielen der Revolution steht.

Im Titel V sind in den Art. 92-97 Aufgaben und Funktionen der Streitkräfte geregelt. Die Uberschrift "Nationale Verteidigung" (defensa nacional) entspricht dem Wortlaut nach den Intentionen des ursprünglichen Regierungsprogramms. Dort wurden als Aufgaben der "neuen nationalen Armee" die Verteidigung des demokratischen Prozesses, der Unabhängigkeit der Nation und der Integrität des Territoriums genannt, also überparteiliche und unabhängige nationale Aufgaben. Art. 93 fügt nunmehr als Aufgabe die Verteidigung der "revolutionären Errungenschaften" hinzu, wobei dieser Begriff den der "Verteidigung des demokratischen Prozesses" ersetzt. In Art. 95 wird die Armee als "sandinistische Volksarmee" (Ejército Popular Sandinista) gekennzeichnet. Damit sind im Verteidigungsbereich zwei wesentliche Abweichungen vom ursprünglichen revolutionären Konzept deutlich: aus der Änderung der Namensbezeichnung resultiert die politische Kontroverse um die Parteilichkeit der Streitkräfte, und die Festlegung auf den von der sandinistischen Partei verantworteten revolutionären Prozeß läßt Zweifel an dem überparteilichen Charakter des Militärs zu. ${ }^{54}$

Im Hinblick auf die Unabhängigkeit der Justiz garantierte das Regierungsprogramm von 1979 dieser das ausschließliche Recht zur Rechtsprechung und verlangte von den Richtern profunde juristische Kenntnisse sowie persönliche Unabhängigkeit als wichtigste Eigenschaften. Diese Grundlagen sind nach der neuen Verfassung nicht mehr gewährleistet. Der Exklusivitätsanspruch ist für die Bereiche Militär (Art. 159 II), Verwaltung (Art. 154) und Staatssicherheitsdienst (Art. 97) nicht gegeben, das heißt sie sind aus dem System der unabhängigen Rechtsprechung ausgeklammert. Die Mitglieder des Obersten Gerichtshofes werden aus einem Dreiervorschlag des Präsidenten von der Nationalversammlung gewählt (Art. 163 I). Der Präsident des Obersten Gerichtshofes wird vom Staatspräsidenten aus dem Richterkollegium ernannt (Art. 163 III). Das neue Rechtsmittel der Normenkontrolle kann nur vom Präsidenten der Republik eingelegt werden sowie vom Präsidenten des Obersten Wahlrates in Angelegenheiten, die in den Bereich seiner Kompetenz fallen, oder wenn sich dazu eine Gruppe von 49 Abgeordneten

53 Vgl. INFORMATIONSDIENST NICARAGUA (IDN) v. 30. 8. 1986, S. 1.

54 Vgl. das Dokument "Solidarität für das nicaraguanische Volk", Beilage zum INFORMATIONSDIENST NICARAGUA (IDN) $4 / 87$ v. 14. 2. 1987. 
der Nationalversammlung bzw. von 30000 Bürgern findet. Diese Voraussetzungen sind unter den gegenwärtigen Machtverhältnissen so gut wie nicht zu erfüllen, was im Grunde einem Verbot, eine Verfassungsbeschwerde einzulegen, gleichkommt. Schließlich ist in diesem Zusammenhang zu erwähnen, daß gemäß Artikel 138 Nr. 2 der Nationalversammlung die authentische Gesetzesinterpretation (Interpretación Auténtica de la Ley) obliegt. Danach verabschiedet das Parlament die Gesetze und nimmt zugleich Kontrollfunktionen wahr, die dem Obersten Gerichtshof zuständen. Diese Beispiele machen deutlich, welch einen Machtzuwachs insbesondere für die Exekutive zu Lasten der Judikative die neue Verfassung bringt.

Die Wirtschaft wird zwar in Artikel 5 (wiederholt in Art. 103) als eine "gemischte Wirtschaftsform" festgeschrieben, die auch privates Eigentum zusichert. Doch wird diese Umsetzung eines der grundlegenden Revolutionsprinzipien von 1979 zu Makulatur angesichts der, nunmehr verfassungsrechtlich abgesicherten, Eingriffsmöglichkeiten des Staates. So lautet zum Beispiel Artikel 99 I: "Der Staat lenkt und plant die nationale Wirtschaft, um die Interessen der Mehrheit zu verteidigen und sie an den Zielen des sozio-ökonomischen Fortschritts zu orientieren." Nach Absatz 2 sowie Artikel 100 und 105 unterstehen dem Staat die Zentralbank, das nationale Finanzsystem einschließlich des Versicherungswesens, der inländische Handel, der gesamte Außenhandel sowie die Investitionspolitik ausländischen Unternehmen gegenüber. Jedes Unternehmen hat sich gemäß Artikel 104 an der staatlichen Wirtschaftspolitik zu orientieren. In Artikel 150 Nr. 13 wird dem Staatspräsidenten persönlich die Wirtschaftslenkung aufgetragen.

Durchgehend fällt an der neuen Verfassung die außerordentlich starke Stellung des Staatspräsidenten auf. Dieser ist gemäß Artikel 144 in Personalunion Staatschef, Regierungschef und oberster Befehlshaber der Streitkräfte. Letzteres auch in Friedenszeiten. Als wichtiger Aspekt ist diesbezüglich zu konstatieren, daß die bewaffneten Kräfte "Fuerzas Armadas Sandinistas" genannt werden, mithin ausgeschlossen sein müßte, daß ein nicht der FSLN angehörender (nichtsandinistischer) Kandidat Präsident und folglich oberster Befehlshaber der "sandinistischen Streitkräfte" werden könnte. Nach Ansicht von Kritikern wird der jetzige Staatspräsident Daniel Ortega damit als eine Art Supergewalt etabliert. ${ }^{55}$ Nicht zuletzt aufgrund der Machtfülle des Präsidenten läßt die Gewaltenteilungsproblematik viele Fragen offen. Die Verfassung erteilt dem Staatspräsidenten gemäß Artikel 150 Nr. 4 die Befugnis, Ausführungsverordnungen mit Gesetzeskraft für den administrativen Bereich und in Bezug auf Angelegenheiten, die im Zusammenhang mit internationalen wirtschaftlichen und politischen Abkommen einschließlich der Auslandsverschuldung stehen, zu erlassen. Er kann gemäß Artikel 185 im Kriegsfall, Katastrophenfall oder wenn es die Sicherheit des Staates verlangt, in der Verfassung festgeschriebene Rechte und Garantien aufheben, muß dies dem Parlament lediglich "zur Kenntnis" geben. Eine Wiederwahl des Präsidenten wird nicht ausgeschlossen, ein gerade für die jungen Demokratien Lateinamerikas ungewöhnliches Faktum. den Verfassern, vgl. Rheinischer Merkur vom 29. 8. 1986, Seite 28. 
Insgesamt ist die neue Verfassung Nicaraguas charakterisiert durch viele demokratische Rechte. Sie ist aber schon wegen nicht konkretisierter Formulierungen, Definitionsproblemen und der durchgängigen, zum Teil nur oberflächlich kaschierten, Identifikation von Staat, Regierung und sandinistischem System angreifbar.

Aus Sicht der Verfasser kann die neue, von der FSLN geprägte Verfassung nicht die Basis für ein authentisches demokratisches System sein, da bereits die institutionellen Voraussetzungen für die Bindung der Staatsgewalt an das Recht aufgehoben sind. Die Verfassungsdiskussion der vergangenen Jahre zeigte einmal mehr, daß diese Revolution auch unter rechtlichen Gesichtspunkten ihren eigenen Ansprüchen nicht gerecht geworden ist. Die Staatspartei FSLN hat die Chance rechtsstaatlicher Entwicklung vertan, die aufgrund umfassender internationaler Hilfestellung nach der Revolution vom 19. Juli 1979 bestand. 
the system is a necessary condition for at least the passive support of the military for the democratic processes. Argentina is in some respects an exception as defeat in the Malvinas/Falklands War of 1982 created an unique situation.

Generally speaking the experiences of dictatorship and exile have led to a reassessment of democracy by Latin American social scientists; the dependence approach of the 1960s has given way to studies on the democratic institutions and the political culture of their countries. However, in view of the precarious economic situation of most of South America the question of whether, despite the present consensus in favour of democracy, democratic government can be consolidated must remain open.

\title{
Costa Rica and its Constitution of 7 November 1949: From the Beginnings to the Present
}

\author{
By Jochen Fuchs
}

The 1949 constitution of Costa Rica is the thirteenth constitutional instrument since the country's declaration of independence from Spain in 1821. During its forty-seven years of existence it has proved a remarkably stable legal foundation of the republic.

Apart from the aspect of constancy this constitution also illustrates the distinctive features of the Costa Rican body politic which have earned the country the epithet of a 'Switzerland of Central Americar.

The author discusses in detail the elements of republicanism and social democracy which have informed the substance of the constitution whose pronounced institutional checks and balances, social basic rights and the proscription of armed forces in peacetime merit particular attention in comparison not only with other Latin American states.

The constitutional order of Costa Rica has from 1949 to the present enjoyed a notably close correspondence of political reality to the legal dispensation of the constitution whereas in many other comparable countries the constitutional framework has atrophied into a mere cloak draped over lawless politics.

\section{The Legal order of Post-Revolutionary Nicaragua: Claims and Reality}

\section{By Marta Patricia Baltodano, Reinhard Junghanns and Michael Sommer}

The basis of this article is the declaration made immediately before the revolution of 19 July 1979 by the Frente Sandinista de Liberacion Nacional (FSLN). In this declaration, the FSLN made a commitment to friendly nations that they would install a democratic form of government, on a foundation of law and order. The first de facto-constitution following the revolution consisted of the Basic Statute (Estatuto Fundamental) enacted 
on 20 July 1979 which prescribes the administrative and organisational policies and procedures of the government, and the statute of 21 August 1979 which outlines the rights and duties of the Nicaraguan citizens. Even during this initial stage it became obvious, from certain formulations in the texts of the statutes, that the Nicaraguan government had no intention of complying with the requirements pertaining to the preservation of fundamental human rights as contained in the international conventions to which Nicaragua also was a signatory beyond that which the government was prepared to concede by virtue of its own domestic legislation.

The first state of emergency was proclaimed in Nicaragua on 22 July 1979 and lasted until 29 April 1980. The introduction of special courts as well as the fluid manner in which the laws were interpreted during this period gave every reason for concern. On 15 March 1982 a state of emergency was again declared and has been in effect to the present time. A short interlude, from the middle of 1984 until 15 October 1985, promised at first to bring about certain improvements but then failed to result in any changes. The hopes that the state of emergency would be lifted with the adoption of the revised constitution on 9 January 1987 were soon disappointed.

In their article the authors furnish evidence that the constitutional development in Nicaragua since the revolution has been marred by numerous institutionalised and partially legalised violations of the law and of the principles of a pluralistic social order. The introduction of the so-called "anti-Somoza peoples courts" (tribunales populares antisomozistas), the laws governing communications media, the laws pertaining to political parties and to elections as well as numerous other rules and regulations make very obvious the discrepancy between the self-imposed standards of the Nicaraguan government and the reality of the revolution and have been the target of criticism from a number of international human rights organizations.

Even the new constitution, mainly inspired by the governing FSLN party, is in the opinion of the authors not the proper foundation upon which an authentic democratic system of government can be built because the institutional prerequisites necessary to bind the authority of the state by law and the legislative processes have been abolished. The constitutional debates during the past years have again shown that the revolutionary government has not, from a legal standpoint, been able to accomplish the tasks it had set itself. The FSLN has wasted the opportunity to develop as a constitutional by based government in Nicaragua, despite the extensive international support it received after the 19 July 1979 revolution. 